\title{
Simulation of Turbo Coded OFDM System
}

\author{
${ }^{1}$ Jyoti Chand, ${ }^{2}$ Deependra Pandey \\ Department of Electronics and Communication Engineering, Amity University, Lucknow
}

\begin{abstract}
The popularity of Orthogonal Frequency Division Multiplexing (OFDM) has been increasing rapidly in a high speed wireless communication systems. In OFDM we basically divide a wideband fading channel into a narrowband channels. OFDM is capable of neglecting or deleting the diverse ( bad) effects of multipath fading with the help of a one tap equalizer. A system is needed that can transmit information wirelessly and accurately. The technique of combining an OFDM which is suitable for high data rate transmission with a Forward Error Correction (FEC) method over a wireless channels have been done already by many engineers. In this thesis, we basically improve the working of an OFDM system by adding Turbo codes. By proper Power allocation and efficient use of coding, the performance at the high data rates improves. Simulation is to be done over two channels- AWGN and Rayleigh channels. In order to improve the performance convolution coding is used [1].
\end{abstract}

Keywords: Orthogonal Frequency Division Multiplexing, Bit Error Rate, Turbo Codes

\section{INTRODUCTION}

A multi carrier modulation technique is used in Orthogonal Frequency Division Multiplexing (OFDM) in which a high rate data stream is divided into multiple low rate data stream and these are further modulated using subcarriers that are orthogonal to each other. There are many advantages of OFDM such as multipath delay spread tolerance and also modulation/demodulation can be done using Fast Fourier Transform (FFT)/ Inverse Fast Fourier Transform (IFFT) that are computationally efficient.

When a single OFDM transmission is in process, all the subcarriers are synchronized to each other in order to restrict the transmission to the digital modulation schemes [2,3].OFDM is considered as a symbol based system in which a large number of a low bit carriers are transmitted in parallel form. A single OFDM is formed by these multi-carriers, hence they are commonly termed as subcarriers.

\section{TURBO CODES}

The addition of Turbo Codes in OFDM System is called as Turbo Coded Orthogonal frequency Division multiplexing (TCOFDM). TCOFDM can yield a significant improvement low energy demand to transmit the data stream [4] [5]. In 1993, Turbo Codes were first presented at the international Conference on Digital communications.

TCOFDM can yield a significant improvement low energy demand to transmit the data stream [4] [5]. In 1993, Turbo Codes were first presented at the international Conference on Digital communications. Until that year, it was widely believed that to achieve near Shannon's bound limit one need to implement a decoder with infinite complexity or close to it. Block Codes or Convolution Codes are useful for implementing a Parallel Concatenated Codes. A trellis structure or state diagram is used at the encoder side and with using a hard decision we decode the data stream required. FANO Algorithm under Sequential decoding is used in this thesis.

\subsection{Turbo Encoding}

The encoder used in a Turbo Codes is a Parallel Concatenated Convolution code $[6,7,8]$. The block diagram of Turbo Encoder is shown in Figure 1. The input binary data sequence is represented by $d k=$ $(d 1, \ldots \ldots . . d N)$. The input sequence is transmitted to the input of a convolution encoder ENC1 and a coded bit stream, $x^{p} \mathrm{k} 1$ is generated. After this, the data sequence is inter leaved, that is, the bits are loaded into a matrix and read out in a way so as to spread the position of the input bits. The bits are taken out in a Pseudo Random Order. The interleaved data sequence is then passed to Second convolution encoder ENC2, and a second coded bit stream and hence $x^{p} \mathrm{k} 2$ is generated. The code sequence that is passed to the modulator for transmission is a multiplexed stream consisting of systematic code bits $x^{s} \mathrm{k}$ and parity bits from both the first encoder $x^{p k 1}$ and the second encoder $x^{p} k 2$. 


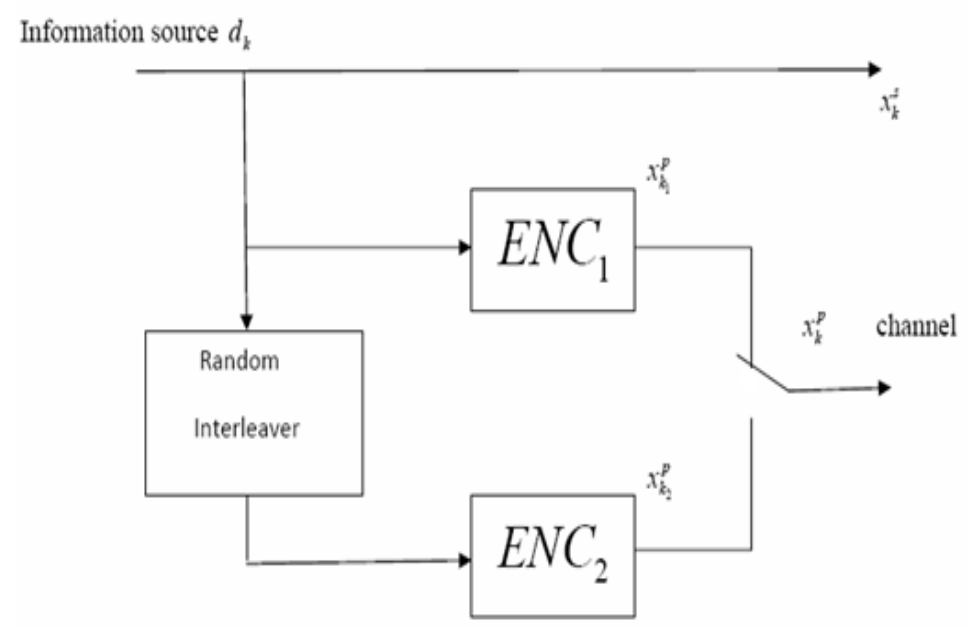

Figure 1- Structure of a Turbo Encoder

\subsection{COMPONENT CODES (RSC)}

The two encoders are recursive systematic convolution codes (RSC) that is a convolution code that uses feedback or in which the un-coded data bits appear in the transmitted code bit sequence.

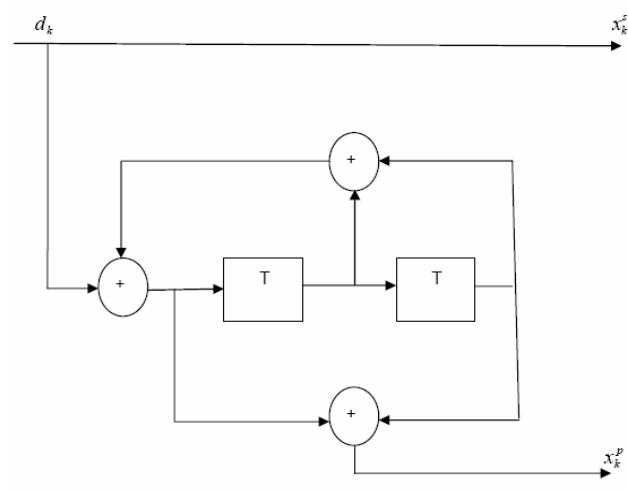

Figure 2- Recursive systematic Convolution Code

The encoder has two output sequence ;

One is the data sequence: $x_{k}^{S}=\left\{x_{1}^{S}, \ldots . . x_{N}^{S}\right\}$,

Other is the parity sequence: $x_{k}^{p}=\left\{x_{1}^{p} \ldots x_{N}^{p}\right\}$.

\subsection{Turbo Decoding}

Block diagram of a turbo decoder is shown in Figure 3.As shown in the diagram the input to the turbo decoder is a sequence of received code values $R_{k}=\left\{y_{k}^{s}, y_{k}^{p}\right\}$ from the demodulator. The turbo decoder consist of a two component decoder-DEC1 to decode sequence from ENC1 and DEC2 to decode sequence from ENC2. The DEC1 takes its input the received sequence systematic values $\left\{y_{k}^{s}\right\}$ and the received sequence parity values $y_{k 1}^{p}$, belonging to the first encoder ENC1. DEC1 output is a sequence of hard estimates EXT1 of the transmitted data is $d_{k}$. Extrinsic data EXT1 does not have any information which is given to DEC2 from DEC1. The information is interleaved and passed to the decoder 2. The inter leaver is same as the encoder in Figure 1. DEC2 takes as its input the (interleaved) systematic received values $y_{k}^{s}$ or the sequence of received parity values $y_{k 2}^{p}$ from the second ENC2, along with the interleaved form of the extrinsic information EXT1 provided by the first encoder ENC1. DEC 2 gives a set of values which when de interleaved (inverse of Inter leaver) constitutes hard estimates EXT2 of the transmitted data sequence $d_{k}$. This extrinsic data is formed without the help of parity bits from which the first encoder is feedback. The process is repeated in a iterative manner. The iterative decoding process adds greatly to the BER performance of turbo codes.DEC2 outputs a value ${ }^{\wedge}\left(d_{k}\right)$, a likelihood representation of the estimate of $d_{k}$.This maximum likelihood value takes the account of transmitting 
a bit ' 0 ' or ' 1 ' based on the systematic and parity information from both component codes. For this we used FANO algorithm under the sequential decoding. We set a particular threshold value and initiate the process accordingly. If the accumulate error crosses or at the threshold level, 'TRACEBACK' is applied in order to minimize the error rate.

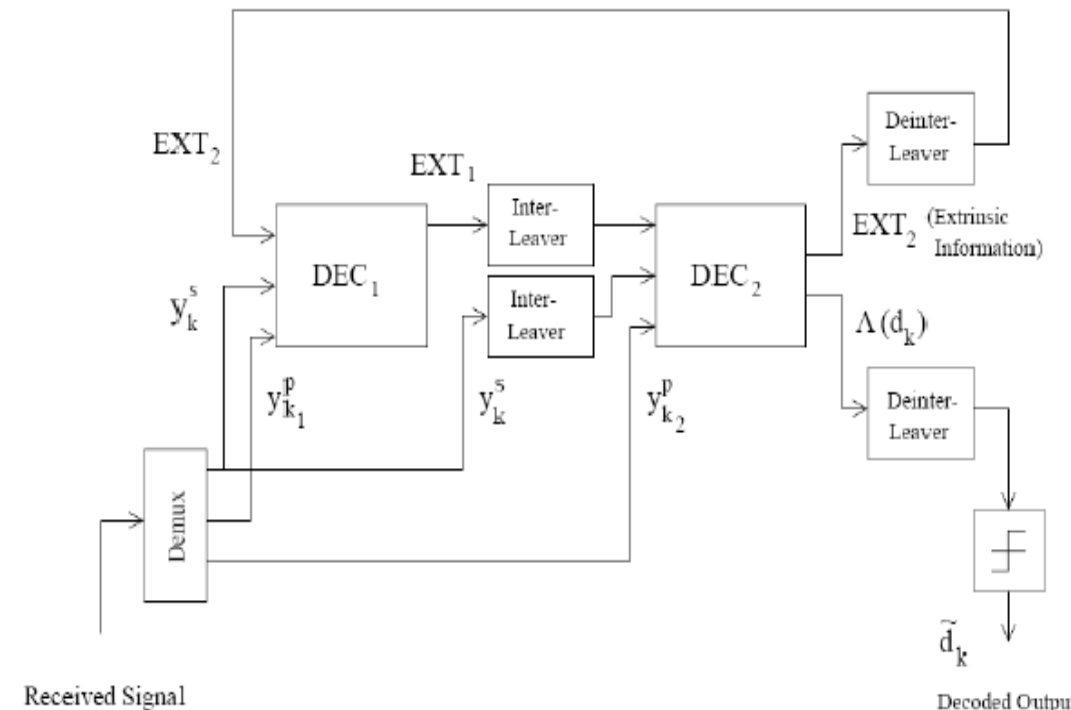

$\mathrm{R}_{\mathrm{k}}=\left\{\mathrm{y}_{\mathrm{k}}^{\mathrm{s}}, \mathrm{y}_{\mathrm{k}}^{\mathrm{p}}\right\}$

Figure 3 - Turbo Decoder

\section{SIMULATION}

3.1 SIMULATION MODEL

The main aim of the research paper is to simulate the OFDM using turbo Codes. The block diagram of entire process is shown in Figure 4.

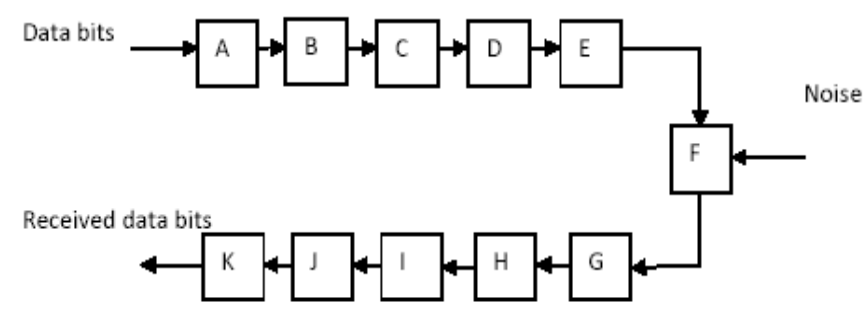

Figure 4- Simulation Model of TCOFDM

Here, $\mathrm{A}=$ turbo encoder, $\mathrm{B}=\mathrm{QAM} / \mathrm{QPSK}$ modulation, $\mathrm{C}=$ Serial to parallel converter, $\mathrm{D}=\mathrm{IIFT}, \mathrm{E}=\mathrm{Parallel}$ To serial converter, $\mathrm{F}=$ Channel with Noise, $\mathrm{G}=$ Serial to parallel converter, $\mathrm{H}=\mathrm{FFT}$, I=Parallel to serial converter, $\mathrm{J}=\mathrm{QAM} / \mathrm{QPSK}$ demodulation and $\mathrm{K}=$ turbo decoder.

\subsection{SIMULATION PARAMETERS}

During simulation, for comparison purpose, the same random messages are generated and for that the radiant function should be in MATLAB.

Table 1 Shows all the parameters used for the simulation of the Turbo coded OFDM. Although these parameters can vary from time to time in order to improve the overall performance of the system. 


\begin{tabular}{|l|l|}
\hline \multicolumn{1}{|c|}{ Parameters } & \multicolumn{1}{c|}{ Values } \\
\hline Digital Modulation & OPSK, DAM \\
\hline Turbo Code Rate & $1 / 3$ \\
\hline Code Generator & 111101 \\
\hline Interleaver Size & $2{ }^{*} 16$ \\
\hline No. of Subearriers & 4 \\
\hline Decoding Algo & Fano Sequential Decoding \\
\hline
\end{tabular}

Table 1 - Simulation Parameters

\section{RESULTS}

Simulations are done to achieve a desired BER. For simulation results, two noise models were considered here: the AWGN and the Rayleigh Channel. The BER performance of the TCOFDM system is compared with the respective un-coded system under the AWGN and Rayleigh channel. "Figure 5" shows the comparison of Un-coded and Turbo coded OFDM with AWGN channel using QAM modulation technique.

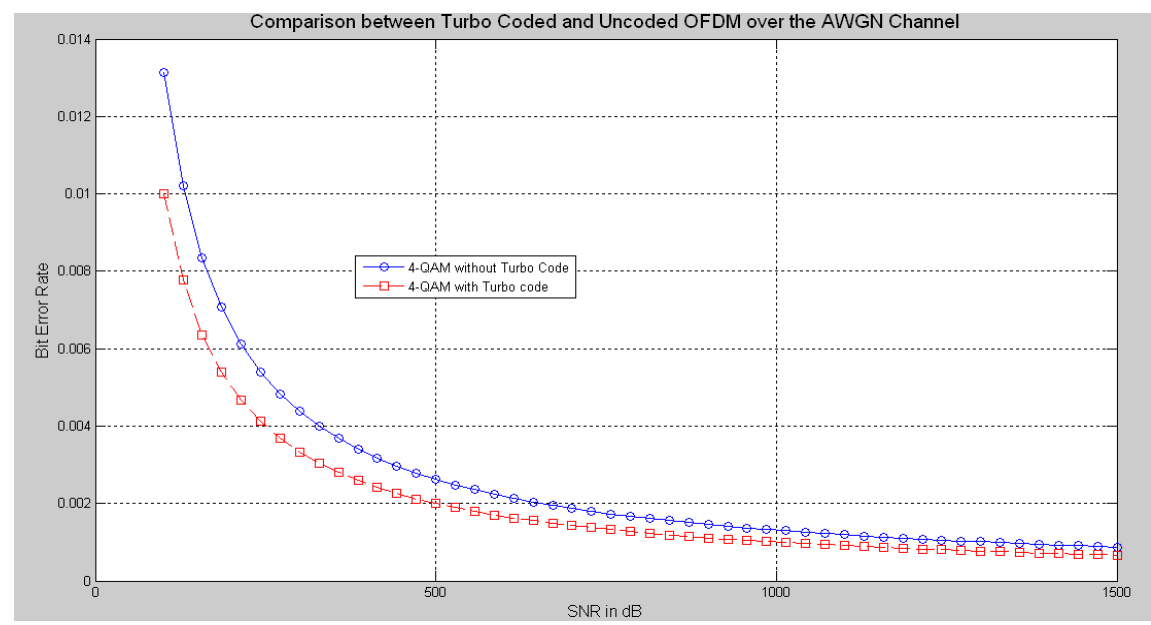

Figure 5 -Comparison between Turbo coded and Un-coded OFDM

Hence in order to improve the performance of OFDM system, turbo code must be added. "Fig 6" shows the BER Vs SNR graph for Un-coded and TC OFDM in which we are using a Rayleigh channel i.e. fading instead of a noisy channel. Here Quadrature Amplitude Modulation is applied.

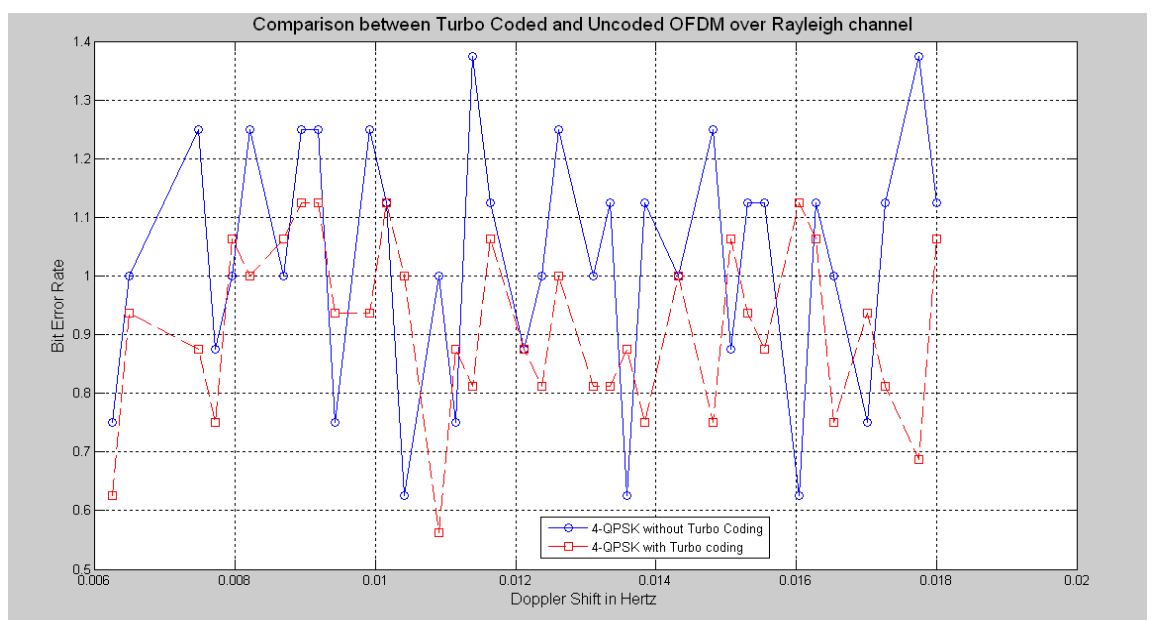

Figure 6- Comparison between Turbo coded and Un-coded OFDM over Rayleigh Channel 
Here, a 4 bit Quadrature Phase Shift Keying is used and the BER of the system is less using Turbo code as compared to Un-coded system. "Figure 7" shows the performance of un-coded and Turbo coded OFDM using QPSK under both a noisy and a faded channel.

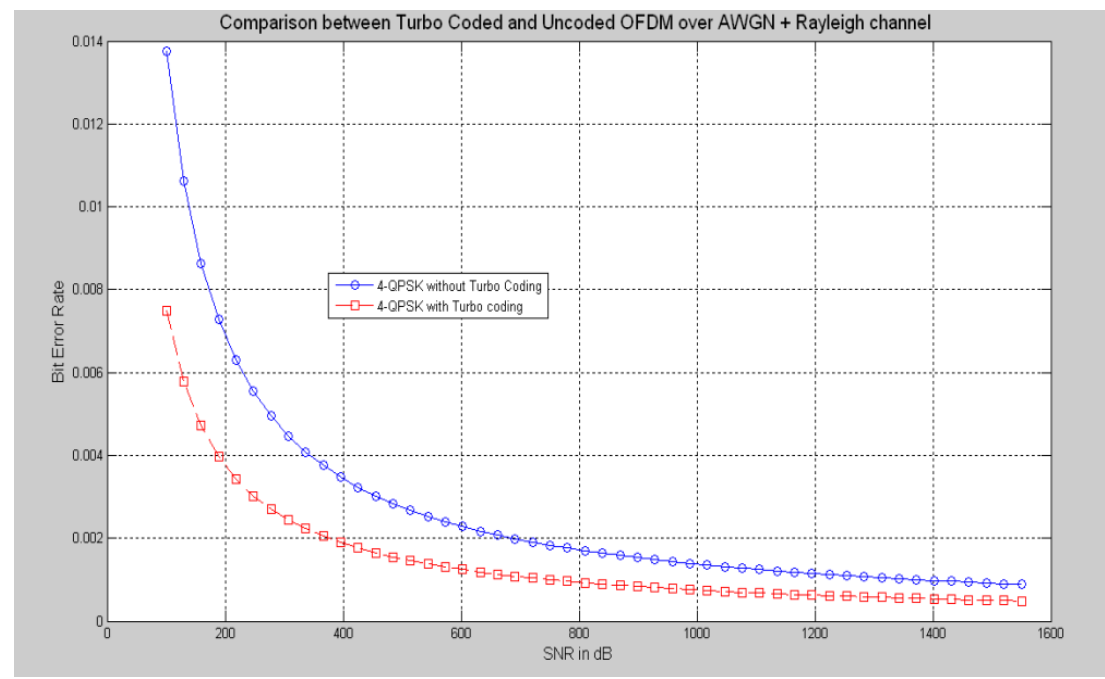

Figure 7- Comparison between Turbo coded and Un-coded OFDM over Rayleigh and AWGN channel

According to the graph the TCOFDM gives much better performance as compared to un-coded system as the error rate of Un-coded OFDM is quite high.

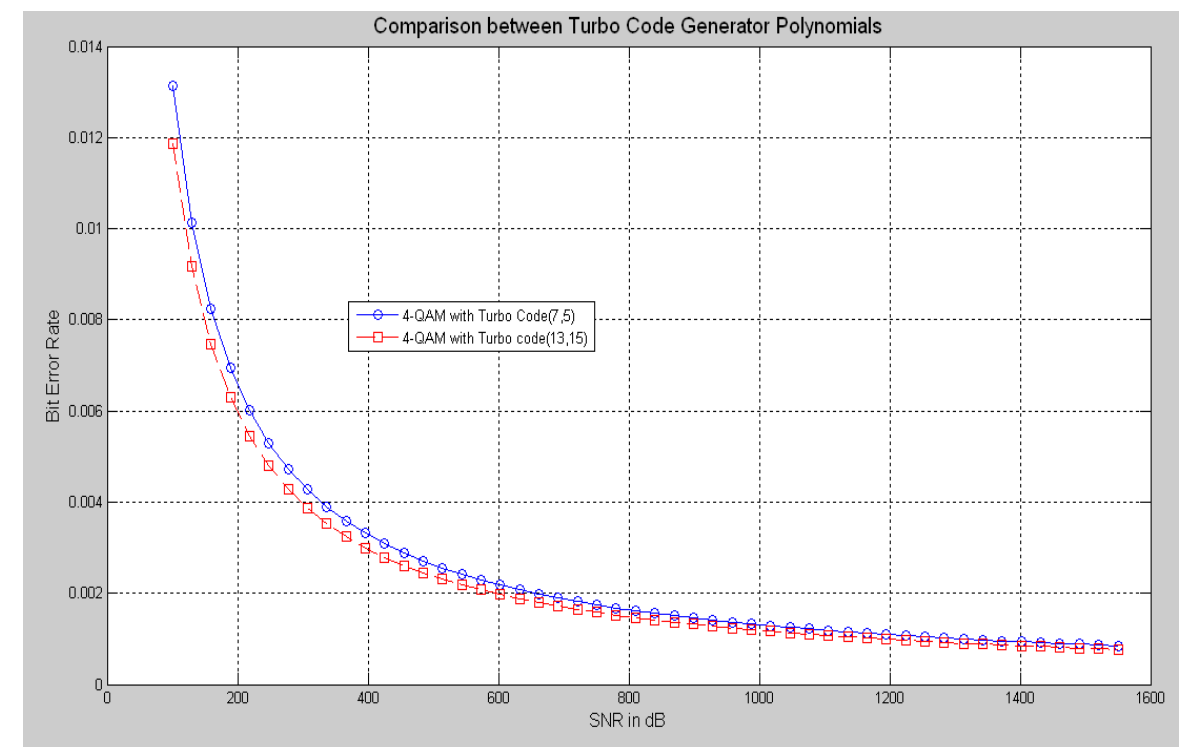

Figure 8- Comparison between different generator polynomials

From the above graph we can say that the performance of TCOFDM for different generator polynomials is quite same. But the BER of $(13,15)$ is slightly better.

\section{CONCLUSION}

To conclude, we can say that Identification of some factors prove that an OFDM system not performing to its potential, hence further implementation is needed in the system. These factor includes Intersymbol Interference (ISI) caused by a dispersive channel, Inter-channel Interference ICI and also the issue of PAPR which is crucial for proper functionality. Several techniques are explored in order to combat some of these problems like by using a Cyclic Prefix and equalization makes the system performance better. Presentation of a result in both AWGN and Rayleigh environments, shows the actual nature or performance of Turbo coded system. The simulation of entire work is done on MATLAB 7.7. First develop an OFDM system model and then apply a Turbo code in order to improve the performance of overall system. The system model developed is quite flexible and can be easily modified or extended to study the performance of this scheme. 


\section{REFERENCES}

[1] M.K. Gupta , Vishwas Sharma "To improve BER of Turbo coded OFDM channel over noisy channel" in Journal of Theoretical and Applied Information Technology @2005-2009 JATIT.

[2] Ramjee Prasad, "OFDM for Wireless Communications systems", Artech House Publishers, 2004.

[3] L. Hanzo , M. Munster, B .J .Choi, T . Keller, "OFDM and MC-CDMA for broadband Multiuser Communications, WLANs and Broadcasting”John Wiley Publishers, 2003.

[4] Lou I Ilunga, Research work on “ Adaptive, Turbo coded OFDM”C 2005.

[5] George White, "Optimised Turbo Codes for Wireless channels". Phd. Thesis communication Research Group Department of Electronics University of York; UK, 10 ${ }^{\text {th }}$ Oct 2001.

[6] C. Berrou, A. Glavieux, and P. Thitimajshima, "Near Shannon Limit Error-correcting Coding: Turbo Codes". Proceedings of the IEEE International Conference on Communications, ICC' 93, Geneva.,pp. 1064-1070, May 1993.

[7] G .D .Forney, “The Viterbi Algorithm”, Proceedings IEEE, vol.61, no.3,pp.268-278, March 1973.

[8] L. Bahl, J. Cocke, F. Jelinek and J. RAviv, “ Optimal Decoding of Linear Codes for Minimising Symbol Error Rate", IEEE Trans. On Information Theory, vol.20.pp.248-287, March 1974.

[9] T.A. Summers and S . G. Wilson, “ SNR Mismatch and Online Estimation in Turbo Decoding”,IEEE Trans. On Communications, vol.46,no.4,pp.421-423, April 1998.

[10] A.G. Burr, G.P.White, "Performance of turbo-Coded OFDM"in IEE Trans. Of International Conference on Universal Personal Communications, 1999. 